\title{
Induction of endoplasmic reticulum stress and unfolded protein response constitutes a pathogenic strategy of group A streptococcus
}

\section{Moshe Baruch ${ }^{1}$, Baruch B. Hertzog ${ }^{1}$, Miriam Ravins ${ }^{1}$, Aparna Anand ${ }^{1}$, Catherine Youting Cheng ${ }^{2}$, Debabrata Biswas ${ }^{1,2}$, Boaz Tirosh ${ }^{3}$ and Emanuel Hanski ${ }^{1,2 *}$}

\footnotetext{
1 Department of Microbiology and Molecular Genetics, Faculty of Medicine, The Hebrew University of Jerusalem (HUJI), Jerusalem, Israel

${ }^{2}$ Department of Microbiology, Center for Research Excellence and Technological Enterprise (CREATE), National University of Singapore (NUS) and NUS-HUJI, Singapore

${ }^{3}$ The School of Pharmacy, Institute for Drug Research, The Hebrew University of Jerusalem, Jerusalem, Israel
}

Edited by:

Eva Medina, Helmholtz Centre for Infection Research, Germany

Reviewed by:

Charlene Kahler, University of

Western Australia, Australia

Yi Xu, Texas A\&M Health Science

Center, USA

\section{${ }^{*}$ Correspondence:}

Emanuel Hanski, Department of Microbiology and Molecular

Genetics, Faculty of Medicine, The Hebrew University of Jerusalem (HUJI), PO Box 12272, Jerusalem 9112102, Israel

e-mail:emanuelh@ekmd.huji.ac.il
The connection between bacterial pathogens and unfolded protein response (UPR) is poorly explored. In this review we highlight the evidence showing that group A streptococcus (GAS) induces endoplasmic reticulum (ER) stress and UPR through which it captures the amino acid asparagine (ASN) from the host. GAS acts extracellularly and during adherence to host cells it delivers the hemolysin toxins; streptolysin O (SLO) and streptolysin S (SLS). By poorly understood pathways, these toxins trigger UPR leading to the induction of the transcriptional regulator ATF4 and consequently to the upregulation of asparagine synthetase (ASNS) transcription leading to production and release of ASN. GAS senses ASN and alters gene expression profile accordingly, and increases the rate of multiplication. We suggest that induction of UPR by GAS and by other bacterial pathogens represent means through which bacterial pathogens gain nutrients from the host, obviating the need to become internalized or inflict irreversible cell damage.

Keywords: group A streptococcus, UPR, metabolism, virulence, asparagine, asparaginase
The endoplasmic reticulum (ER) is an essential organelle that controls protein and lipid biosynthesis, protein folding and trafficking and calcium homeostasis (Berridge, 2002). Different perturbations at the cellular level can affect ER homeostasis inducing the accumulation of misfolded proteins within the ER lumen or changing its lipid composition. These processes ultimately lead to ER stress. To alleviate these conditions, the ER launches the unfolded protein response (UPR), allowing the cells to adapt to the environmental stresses and survive (Walter and Ron, 2011). However, under prolonged stress conditions, when stresses remain unmitigated, UPR triggers programmed cell death (Tabas and Ron, 2011; Woehlbier and Hetz, 2011).

In mammalian cells UPR is mediated by three major signal transduction pathways: PKR-like endoplasmic reticulum kinase (PERK), inositol-requiring enzyme 1 (IRE1), and activating transcription factor 6 (ATF6) (Figure 1). These signaling pathways are all initiated when misfolded proteins are sensed in the ER lumen. These three pathways combat ER stress through complementary strategies including: (a) attenuation of global protein translation to reduce the influx of client proteins into the ER; (b) up-regulation of chaperones and enzymes involved in refolding of misfolded proteins; and (c) enhancing ER-associated degradation (ERAD) to facilitate clearance of misfolded proteins from the ER (Schroder and Kaufman, 2005; Ron and Walter, 2007). As mentioned above, a variety of external stimuli have been shown to cause UPR. This includes abiotic stresses; pharmacological agents and toxins producing imbalance of ER calcium and redox; anti-inflammatory agents causing vigorous protein synthesis; energy deprivation, amino acids and ATP depletion, genetic mutations occurring in protein misfolding diseases and microbial pathogens (Schroder and Kaufman, 2005; Yoshida, 2007; Walter and Ron, 2011). Since there are several potential crosstalks between UPR and microbial sensing pathways that trigger immune responses (Hotamisligil, 2010; Hasnain et al., 2012; Hetz, 2012; Martinon, 2012; Claudio et al., 2013), investigators started to explore how microbial pathogens cope with UPR that they induce and whether or not they are able to exploit UPR for their own "benefit" (Roy et al., 2006).

Under viral infection these questions seem most relevant as viruses cause both cell stress through their replication and by over-expressing viral proteins (Zhang and Wang, 2012; Claudio et al., 2013; Hare and Mossman, 2013). Indeed, many viruses have evolved strategies to ensure completion of their infectious cycle by actively interfering with host translation shut-down and prolonging infected-cells life span, despite severe stress conditions (He, 2006; Roy et al., 2006; Martinon, 2012). Moreover, viruses developed strategies to differentially regulate the three UPR signaling pathways (Figure 1) and to dampen downstream inflammatory responses, and thus exploit ER stress for their own benefits (He, 2006; Roy et al., 2006; Martinon, 2012; Stahl et al., 2013).

Much less is known about the role of UPR in bacterial infections. Indeed, it was reported that the pathogen itself as well as bacterial toxins both induce UPR. Yet, it remained elusive whether UPR induction represents a genuine pathogenic 


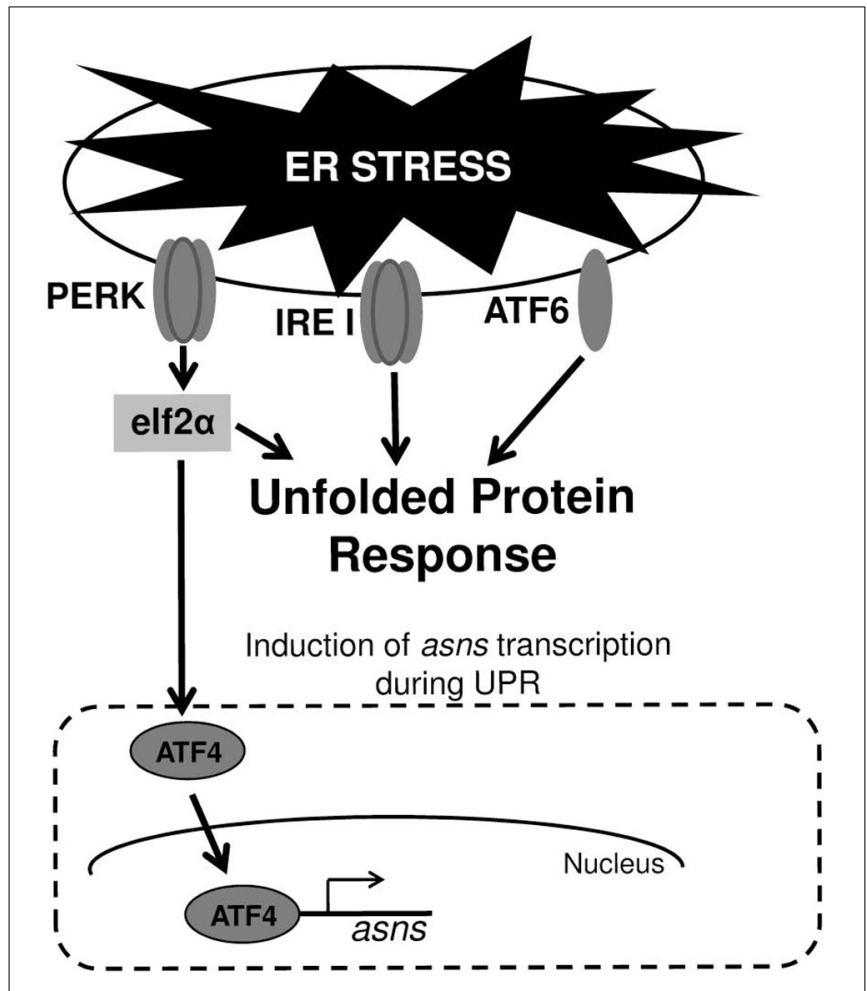

FIGURE 1 | ER stress and UPR. Upper panel-the three transmembrane receptor proteins that are responsible for triggering UPR in cells experiencing ER stress. Lower panel-induction of asns transcription through the PERK/elF2/ATF4 pathway. During UPR, PERK phosphorylates elf2 $\alpha$, which in turn elevates the translation of the transcription factor ATF4. ATF4 upregulates the transcription of several genes including that of asns.

trait of the bacteria, constitutes a pathway through which the host mounts immune defenses or is just a vicissitude of the interaction. For example, shiga toxins (Stxs) are genetically and structurally related cytotoxins expressed by the enteric pathogens Shigella dysenteriae serotype 1 and an expanding number of Shiga toxin-producing Escherichia coli. Following retrograde transport Stxs are translocated into the ER lumen and then the active fragment is translocated across the ER membrane to reach the cytoplasm where it de-purinates the $28 \mathrm{~S}$ rRNA subunit of the ribosome. This in turn, triggers UPR and leads to downstream signaling through the p38 mitogen-activated protein kinases (MAPK) cascades (Liang et al., 2006), which appear to be critical for activation of innate immunity and regulation of apoptosis (Tesh, 2012). Cholera toxin (CT) is a major virulence factor of Vibrio cholera that reaches the lumen of the ER in a similar way to that of Stxs (Sandvig et al., 1992). In the ER lumen, CT unfolds and the A1 chain interacts with IRE1 to initiate UPR. The unfolded A1 chain co-opts the ER to retro-transport itself by the ERAD machinery into the cytosol, where it refolds, escapes degradation and becomes catalytically active. In addition, an inflammatory response is generated by the activated IRE1 RNase. This RNase degrades cellular RNAs that are detected by the retinoic-acid inducible gene 1 (RIG-1), a cytosolic sensor of RNA viruses. This in turn activates the NF- $\kappa$ B and interferon pathways (Cho et al., 2013).
The ability to induce UPR is not limited only to Stxs and CT, but also exists for pore-forming toxins (PFTs) that constitute the largest class of bacterial toxins and are produced by the most clinically important bacterial pathogens. In Caenorhabditis elegans infected with bacteria expressing PFTs, UPR is induced and lose of ATF6 and IRE1 pathways (Figure 1) by genetic manipulations leads to hypersensitivity of the nematode to attack by PFT-producing bacteria. These findings suggest that ER homeostasis or induction of immune response via ER-signaling protects the host against these toxins (Bischof et al., 2008). Brucella melitensis is a facultative intracellular bacterium that fuses with the ER to replicate. This results in a marked reorganization of the ER around the replicating bacteria and triggering of UPR. UPR induction requires both live bacteria and the expression of a specific Brucella protein (Smith et al., 2013). Another facultative intracellular pathogen, Listeria monocytogenes, induces ER expansion and UPR prior to its entry into host cells. Its mutant, deficient of the PFT, listeriolysin O (LLO), is unable to induce UPR. Furthermore, induction of UPR by ER-stressors before infection with L. monocytogenes reduces bacterial intracellular loads, suggesting that UPR may represent a defense response of the host against L. monocytogenes infection (Pillich et al., 2012). The first indication that UPR induction by a bacterial pathogen could be a virulence strategy was reported for GAS. Cywes-Bentley and colleagues demonstrated that infection of keratinocyte by GAS deregulates intracellular calcium through the action of the PFT, protein- SLO. This in turn causes UPR, subsequently leading to loss of epithelial integrity, cell detachment and apoptosis (Cywes Bentley et al., 2005).

GAS is an obligate human pathogen and the fourth most common bacterial cause of human mortality (Carapetis et al., 2005). GAS causes a vast array of human manifestations ranging from mild infections such as pharyngitis and impetigo to highly invasive and life-threatening infections such as necrotizing fasciitis and toxic shock, as well as to the autoimmune syndromes rheumatic fever and glomerulonephritis (Cunningham, 2000; Walker et al., 2014). SLO and SLS are essential virulence factors of GAS as was demonstrated both in ex-vivo and in-vivo studies (Walker et al., 2014). SLO is a PFT belonging to the family of cholesterol-dependent cytolysins (CDCs) produced by several pathogenic Gram-positive bacteria including Streptococcus, Clostridium, and Listeria species. CDCs share many features including, a similar overall molecular structure, mechanisms of membrane recognition and pore formation (Hotze and Tweten, 2012). SLO is co-expressed with GAS NAD-glycohydrolase (SPN) and SLO-mediated translocation of SPN has been shown to be an additional way by which this toxin contributes to GAS virulence (Madden et al., 2001; Bricker et al., 2002). Another toxin with which SLO acts in concert during GAS infections is SLS (Ginsburg and Kohen, 1995; Fontaine et al., 2003; Watanabe et al., 2013). SLS is a small, ribosomally produced bacteriocin-like toxin that undergoes heterocyclic modifications at specific residues to confer activity. As SLO, SLS-like peptides are produced by some streptococci and other Gram-positive pathogens as Clostridia, Listeria, and Staphylococci species (Molloy et al., 2011). Finally, both SLO and SLS are delivered into host cells more efficiently by adhering bacteria compared to non-adhering bacteria, thus close contact 
of the bacteria to the cell promotes efficient delivery of the toxins (Ofek et al., 1990; Ruiz et al., 1998).

Although GAS has been considered as an extracellular pathogen, studies from various laboratories have shown that the bacterium has the propensity to invade different cell types in vitro (Courtney et al., 2002). This capacity was suggested to contribute to GAS persistence within the host. Indeed, GAS was cultivable from surgical specimens of human tonsils, even after treatment of the excised tissue with antibiotics (Osterlund et al., 1997). Since GAS does not proliferate within mammalian cells, the significance of GAS intracellular phase was not explored in depth in in vivo models of human infections. However, internalization of GAS via the clathrin-dependent pathway was reported to be inhibited by low doses of SLO when the latter was produced extracellularly, whereas intracellularly produced SLO protected GAS from various modes of intracellular killing (Logsdon et al., 2011; O'Seaghdha and Wessels, 2013).

Recently it was discovered that GAS induces UPR to capture ASN from the host (Baruch et al., 2014). This was found while investigating the conditions under which the GAS quorum sensing locus sil is self-activated. sil is situated on an mobile genetic element that may have been acquired before GAS speciation and remained present in about $20 \%$ of GAS clinical isolates and is widely prevalent in the GAS genetically close relative Streptococcus dysgalactiae subsp. equisimilis (Belotserkovsky et al., 2009). In the GAS M14 serotype, sil controls virulence as was shown using different animal models of human NF (HidalgoGrass et al., 2002, 2004; Kizy and Neely, 2009). Hitherto, it was possible to activate sil by providing the bacterium with a minute quantity of the mature synthetic autoinducer peptide SilCR (Belotserkovsky et al., 2009), but the conditions under which sil is naturally self-activated were not identified. Later, it was discovered that sil is temporarily self-activated in vivo, during the initial stages of soft-tissue infection in a murine model of human NF. Furthermore, it was discovered that sil is also activated ex vivo upon adherence to various types of eukaryotic cells (Baruch et al., 2014).

Cyews-Bently et al. showed that GAS induces SLO-mediated ER-stress at low multiplicity of infection (MOI) of keratinocyte cells due to dysregulation of intracellular calcium (Cywes Bentley et al., 2005). In accordance with these findings, it was shown that sil activation occurred at low MOI of intact but not lysed eukaryotic cells, did not required internalization of GAS and was mediated by delivery of SLO and SLS toxins (Baruch et al., 2014). To delineate the cellular process that is triggered by SLO and SLS delivery the involvement of autophagy, apoptosis, and necrosis that are affected by the hemolysin toxins and were shown to be linked to GAS pathogenesis was examined (Baruch et al., 2014). Using mutated mouse embryonic fibroblast cells (MEFs) in combination with various pharmacological agents, the involvement of the indicated cellular processes in sil activation were ruled out. The facts that host cell intactness was essential to observe sil activation and that hemolysin toxins were involved, together with the report that SLO triggers ER stress (Cywes Bentley et al., 2005), hinted at the involvement of the latter process. Indeed, induction of UPR using the ER stressors thapsigargin (TG), and dithiothreitol (DTT) produced a conditioned media capable of activating sil. Furthermore, addition of TG to MEFs-infected by GAS accelerated sil activation (Baruch et al., 2014). During the testing of different eukaryotic cells for the ability to activate sil, it was discovered that ASN per-se is responsible for sil activation (Baruch et al., 2014). This finding together with the fact that asns transcription of host cells is strongly upregulated during UPR through the PERK-eIF2-ATF4 pathway (Figure 1) (Balasubramanian et al., 2013), led to the examination of asns transcription during MEFs infection by GAS. As predicted, it was found that there is a significant increase in asns transcription in GAS-infected MEFs that is dependent on SLO and SLS (Baruch et al., 2014). Taken together, these results supported the model in which delivery of SLO and SLS toxins from GAS to eukaryotic cells during GAS adherence generates ER stress. This in turn leads to UPR, enhanced production of the response regulator ATF4, activation of ASNS and release of ASN to the medium, through a mechanism yet unknown (Figure 2). ASN is than sensed by GAS to activate sil.

To corroborate this model, asparaginase (ASNase), which is widely used as a chemotherapeutic agent (Pui et al., 2008) was tested for its ability to inhibit sil activation. Indeed, ASNase obliterated sil activation ex vivo and in vivo, but most fascinatingly, also arrested GAS growth (Baruch et al., 2014). It was found that ASNase inhibits the growth of GAS irrespective of the serotype and presence or absence of sil (Figure 2). Therefore, it was decided to profile by RNA sequencing (RNA-seq) the RNA expression, in the globally disseminated highly invasive M1T1 GAS clone, (Maamary et al., 2012), after the addition of

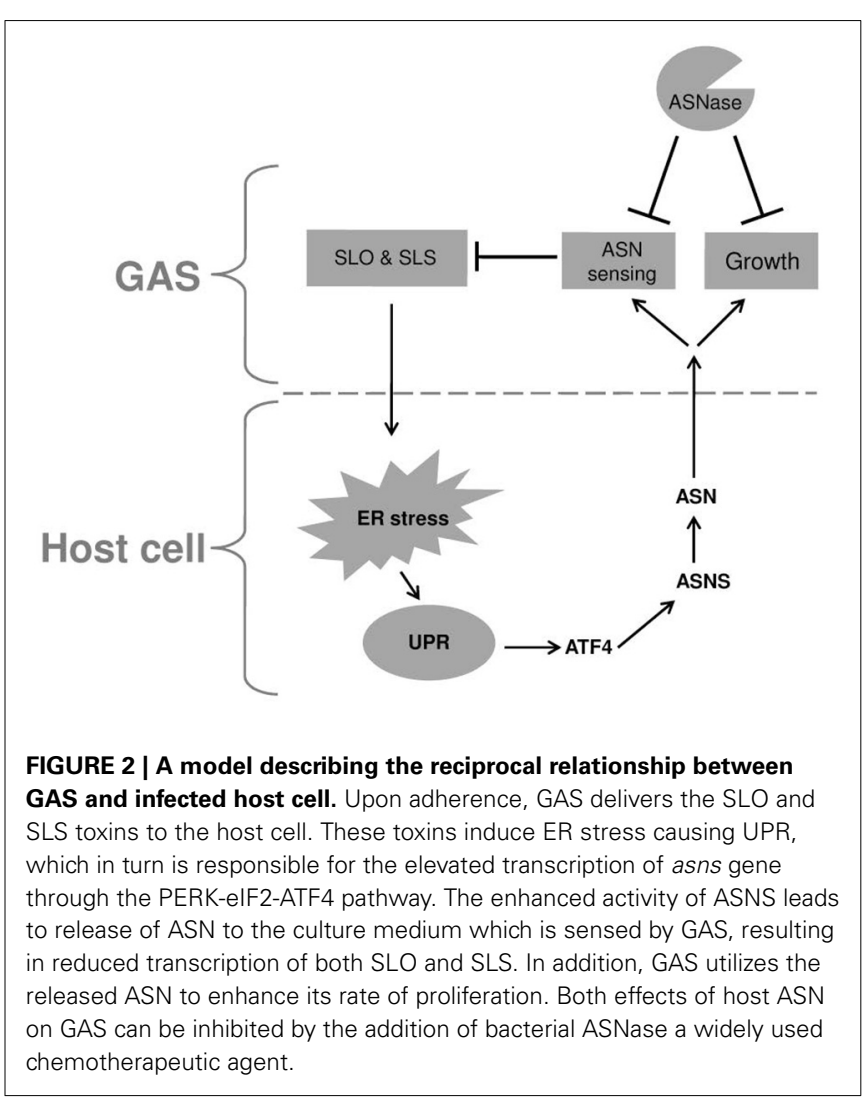


ASNase. It was found that $16.7 \%$ of GAS genes had a significantly altered expression in the absence vs. the presence of ASN (Baruch et al., 2014). Among others, the transcription of genes involved in GAS replication such as polA and lig was down regulated in ASN absence, while the transcription of genes encoding SLO and SLS were upragulated (Baruch et al., 2014 and Figure 2). Most importantly, testing the ability of ASNase to prevent GAS bacteremia showed that ASNase prevented GAS proliferation in whole human blood and in a murine model of human GAS bacteremia (Baruch et al., 2014).

It is suggested that the above-mentioned mechanism of GAS to gain ASN from the host is a central feature in its pathogenesis. This notion is supported by the findings that GAS involves SLO and SLS in this process. These two toxins are considered to constitute key virulence factors of GAS and are involved in many of GAS manifestations (Walker et al., 2014). Moreover, both SLO and SLS levels of transcription are strongly augmented by the absence of ASN (Baruch et al., 2014 and Figure 2). This finding suggests that ASN-mediated sensing of the host by GAS allows the bacterium to regulate the production of its main two toxins, in order to reach a level that on one hand permits GAS to stress the host but on the other hand to maintain homeostasis and avoid inflicting an irreversible damage. This trait is sustained mainly because GAS stresses the host ER, which has an intrinsic capacity to alleviate a wide range of stresses by mounting the UPR response (Schroder and Kaufman, 2005; Zhang and Kaufman, 2006; Ron and Walter, 2007; Walter and Ron, 2011).

The concentration of released ASN allows GAS to assess its population size that is in close contact with the host as well as the host stress status, i.e., whether or not it could sustain more stress to release even more nutrients without progressing into irreversible cell death. Consequently, these assessments may be used by GAS to regulate the level of virulence factors expression, and avoid their metabolically costly production before reaching a critical mass that ensures the successful mounting of invasive infection. Indeed it was found using the murine model of soft-tissue infection, that sil, which serves as a reporter for ASN sensing, is activated transiently at the very early steps of the infection, way before GAS disseminates into internal organs (Baruch et al., 2014).

It was observed that low levels of SLO inhibit GAS internalization by human keratinocytes (Logsdon et al., 2011). Intriguingly, it was reported that the facultative intracellular pathogen L. monocytogenes induces UPR through its LLO toxin (which shares $58.0 \%$ amino acids similarity with SLO) when present extracellularly. This in turn reduces the level of intracellular bacteria, due to the triggering of host defense responses (Pillich et al., 2012). It is speculated that L. monocytogenes regulates LLO expression also upon sensing of host metabolites that are released upon UPR induction. Finally, it was reported that Mycobacterium tuberculosis, which induces ER stress in granulomas during infection in humans (Seimon et al., 2010), exploits host ASN to assimilate nitrogen and resist acid stress during infection (Gouzy et al., 2014). Similar mechanisms of nitrogen assimilation were reported for Helicobacter pylori (Shibayama et al., 2011) and Campylobacter jejuni (Hofreuter et al., 2008). In summary, these studies emphasize the tight connection that has evolved during evolution between physiology and virulence. Understanding of this connection at the molecular level should pave the way for development of new ways to control severe human infectious diseases.

\section{REFERENCES}

Balasubramanian, M. N., Butterworth, E. A., and Kilberg, M. S. (2013). Asparagine synthetase: regulation by cell stress and involvement in tumor biology. Am. J. Physiol. Endocrinol. Metab. 304, E789-E799. doi: 10.1152/ajpendo. 00015.2013

Baruch, M., Belotserkovsky, I., Hertzog, B. B., Ravins, M., Dov, E., McIver, K. S., et al. (2014). An extracellular bacterial pathogen modulates host metabolism to regulate its own sensing and proliferation. Cell 156, 97-108. doi: 10.1016/j.cell.2013.12.007

Belotserkovsky, I., Baruch, M., Peer, A., Dov, E., Ravins, M., Mishalian, I., et al. (2009). Functional analysis of the quorum-sensing streptococcal invasion locus (sil). PLoS Pathog. 5:e1000651. doi: 10.1371/journal.ppat.1000651

Berridge, M. J. (2002). The endoplasmic reticulum: a multifunctional signaling organelle. Cell Calcium 32, 235-249. doi: 10.1016/S0143416002001823

Bischof, L. J., Kao, C. Y., Los, F. C., Gonzalez, M. R., Shen, Z., Briggs, S. P., et al. (2008). Activation of the unfolded protein response is required for defenses against bacterial pore-forming toxin in vivo. PLoS Pathog. 4:e1000176. doi: 10.1371/journal.ppat.1000176

Bricker, A. L., Cywes, C., Ashbaugh, C. D., and Wessels, M. R. (2002). NAD+glycohydrolase acts as an intracellular toxin to enhance the extracellular survival of group A streptococci. Mol. Microbiol. 44, 257-269. doi: 10.1046/j.13652958.2002.02876.x

Carapetis, J. R., Steer, A. C., Mulholland, E. K., and Weber, M. (2005). The global burden of group A streptococcal diseases. Lancet Infect. Dis. 5, 685-694. doi: 10.1016/S1473-3099(05)70267-X

Cho, J. A., Lee, A. H., Platzer, B., Cross, B. C., Gardner, B. M., De Luca, H., et al. (2013). The unfolded protein response element IRElalpha senses bacterial proteins invading the ER to activate RIG-I and innate immune signaling. Cell Host Microbe 13, 558-569. doi: 10.1016/j.chom.2013.03.011

Claudio, N., Dalet, A., Gatti, E., and Pierre, P. (2013). Mapping the crossroads of immune activation and cellular stress response pathways. EMBO J. 32, 1214-1224. doi: 10.1038/emboj.2013.80

Courtney, H. S., Hasty, D. L., and Dale, J. B. (2002). Molecular mechanisms of adhesion, colonization, and invasion of group A streptococci. Ann. Med. 34, 77-87. doi: 10.1080/07853890252953464

Cunningham, M. W. (2000). Pathogenesis of group A streptococcal infections. Clin. Microbiol. Rev. 13, 470-511. doi: 10.1128/CMR.13.3.470-511.2000

Cywes Bentley, C., Hakansson, A., Christianson, J., and Wessels, M. R. (2005). Extracellular group A Streptococcus induces keratinocyte apoptosis by dysregulating calcium signalling. Cell. Microbiol. 7, 945-955. doi: 10.1111/j.14625822.2005.00525.x

Fontaine, M. C., Lee, J. J., and Kehoe, M. A. (2003). Combined contributions of streptolysin $\mathrm{O}$ and streptolysin $\mathrm{S}$ to virulence of serotype M5 Streptococcus pyogenes strain Manfredo. Infect. Immun. 71, 3857-3865. doi: 10.1128/IAI.71.7.3857-3865.2003

Ginsburg, I., and Kohen, R. (1995). Synergistic effects among oxidants, membranedamaging agents, fatty acids, proteinases, and xenobiotics: killing of epithelial cells and release of arachidonic acid. Inflammation 19, 101-118. doi: 10.1007/BF01534384

Gouzy, A., Larrouy-Maumus, G., Bottai, D., Levillain, F., Dumas, A., Wallach, J. B., et al. (2014). Mycobacterium tuberculosis exploits asparagine to assimilate nitrogen and resist acid stress during infection. PLoS Pathog. 10:e1003928. doi: 10.1371/journal.ppat. 1003928

Hare, D., and Mossman, K. L. (2013). Novel paradigms of innate immune sensing of viral infections. Cytokine 63, 219-224. doi: 10.1016/j.cyto.2013.06.001

Hasnain, S. Z., Lourie, R., Das, I., Chen, A. C., and McGuckin, M. A. (2012). The interplay between endoplasmic reticulum stress and inflammation. Immunol. Cell Biol. 90, 260-270. doi: 10.1038/icb.2011.112

He, B. (2006). Viruses, endoplasmic reticulum stress, and interferon responses. Cell Death Differ. 13, 393-403. doi: 10.1038/sj.cdd.4401833

Hetz, C. (2012). The unfolded protein response: controlling cell fate decisions under ER stress and beyond. Nat. Rev. Mol. Cell Biol. 13, 89-102. doi: $10.1038 / \mathrm{nrm} 3270$ 
Hidalgo-Grass, C., Dan-Goor, M., Maly, A., Eran, Y., Kwinn, L. A., Nizet, V., et al. (2004). Effect of a bacterial pheromone peptide on host chemokine degradation in group A streptococcal necrotising soft-tissue infections. Lancet 363, 696-703. doi: 10.1016/S0140-6736(04)15643-2

Hidalgo-Grass, C., Ravins, M., Dan-Goor, M., Jaffe, J., Moses, A. E., and Hanski, E. (2002). A locus of group A Streptococcus involved in invasive disease and DNA transfer. Mol. Microbiol. 46, 87-99. doi: 10.1046/j.1365-2958.2002.03127.x

Hofreuter, D., Novik, V., and Galan, J. E. (2008). Metabolic diversity in Campylobacter jejuni enhances specific tissue colonization. Cell Host Microbe 4, 425-433. doi: 10.1016/j.chom.2008.10.002

Hotamisligil, G. S. (2010). Endoplasmic reticulum stress and the inflammatory basis of metabolic disease. Cell 140, 900-917. doi: 10.1016/j.cell.2010.02.034

Hotze, E. M., and Tweten, R. K. (2012). Membrane assembly of the cholesteroldependent cytolysin pore complex. Biochim. Biophys. Acta 1818, 1028-1038. doi: 10.1016/j.bbamem.2011.07.036

Kizy, A. E., and Neely, M. N. (2009). First Streptococcus pyogenes signature-tagged mutagenesis screen identifies novel virulence determinants. Infect. Immun. 77, 1854-1865. doi: 10.1128/IAI.01306-08

Liang, S. H., Zhang, W., McGrath, B. C., Zhang, P., and Cavener, D. R. (2006). PERK (eIF2alpha kinase) is required to activate the stress-activated MAPKs and induce the expression of immediate-early genes upon disruption of ER calcium homoeostasis. Biochem. J. 393, 201-209. doi: 10.1042/BJ20050374

Logsdon, L. K., Hakansson, A. P., Cortes, G., and Wessels, M. R. (2011). Streptolysin $\mathrm{O}$ inhibits clathrin-dependent internalization of group A Streptococcus. MBio 2:e00332-e00310. doi: 10.1128/mBio.00332-10

Maamary, P. G., Ben Zakour, N. L., Cole, J. N., Hollands, A., Aziz, R. K., Barnett, T. C., et al. (2012). Tracing the evolutionary history of the pandemic group A streptococcal M1T1 clone. FASEB J. 26, 4675-4684. doi: 10.1096/fj.12-212142

Madden, J. C., Ruiz, N., and Caparon, M. (2001). Cytolysin-mediated translocation (CMT): a functional equivalent of type III secretion in gram-positive bacteria. Cell 104, 143-152. doi: 10.1016/S0092-8674(01)00198-2

Martinon, F. (2012). The endoplasmic reticulum: a sensor of cellular stress that modulates immune responses. Microbes Infect. 14, 1293-1300. doi: 10.1016/j.micinf.2012.07.005

Molloy, E. M., Cotter, P. D., Hill, C., Mitchell, D. A., and Ross, R. P. (2011). Streptolysin S-like virulence factors: the continuing sagA. Nat. Rev. Microbiol. 9, 670-681. doi: 10.1038/nrmicro2624

Ofek, I., Zafriri, D., Goldhar, J., and Eisenstein, B. I. (1990). Inability of toxin inhibitors to neutralize enhanced toxicity caused by bacteria adherent to tissue culture cells. Infect. Immun. 58, 3737-3742.

O'Seaghdha, M., and Wessels, M. R. (2013). Streptolysin O and its co-toxin NADglycohydrolase protect group A Streptococcus from Xenophagic killing. PLoS Pathog. 9:e1003394. doi: 10.1371/journal.ppat.1003394

Osterlund, A., Popa, R., Nikkila, T., Scheynius, A., and Engstrand, L. (1997). Intracellular reservoir of Streptococcus pyogenes in vivo: a possible explanation for recurrent pharyngotonsillitis. Laryngoscope 107, 640-647. doi: 10.1097/00005537-199705000-00016

Pillich, H., Loose, M., Zimmer, K. P., and Chakraborty, T. (2012). Activation of the unfolded protein response by Listeria monocytogenes. Cell. Microbiol. 14, 949-964. doi: 10.1111/j.1462-5822.2012.01769.x

Pui, C. H., Robison, L. L., and Look, A. T. (2008). Acute lymphoblastic leukaemia. Lancet 371, 1030-1043. doi: 10.1016/S0140-6736(08)60457-2

Ron, D., and Walter, P. (2007). Signal integration in the endoplasmic reticulum unfolded protein response. Nat. Rev. Mol. Cell Biol. 8, 519-529. doi: $10.1038 / \mathrm{nrm} 2199$

Roy, C. R., Salcedo, S. P., and Gorvel, J. P. (2006). Pathogen-endoplasmic-reticulum interactions: in through the out door. Nat. Rev. Immunol. 6, 136-147. doi: 10.1038/nril775

Ruiz, N., Wang, B., Pentland, A., and Caparon, M. (1998). Streptolysin O and adherence synergistically modulate proinflammatory responses of keratinocytes to group A streptococci. Mol. Microbiol. 27, 337-346. doi: 10.1046/j.13652958.1998.00681.x

Sandvig, K., Garred, O., Prydz, K., Kozlov, J. V., Hansen, S. H., and Van Deurs, B. (1992). Retrograde transport of endocytosed Shiga toxin to the endoplasmic reticulum. Nature 358, 510-512. doi: 10.1038/ $358510 \mathrm{a} 0$

Schroder, M., and Kaufman, R. J. (2005). ER stress and the unfolded protein response. Mutat. Res. 569, 29-63. doi: 10.1016/j.mrfmmm.2004.06.056

Seimon, T. A., Kim, M. J., Blumenthal, A., Koo, J., Ehrt, S., Wainwright, H., et al. (2010). Induction of ER stress in macrophages of tuberculosis granulomas. PLoS ONE 5:e12772. doi: 10.1371/journal.pone.0012772

Shibayama, K., Takeuchi, H., Wachino, J., Mori, S., and Arakawa, Y. (2011). Biochemical and pathophysiological characterization of Helicobacter pylori asparaginase. Microbiol. Immunol. 55, 408-417. doi: 10.1111/j.1348-0421.2011.00333.x

Smith, J. A., Khan, M., Magnani, D. D., Harms, J. S., Durward, M., Radhakrishnan, G. K., et al. (2013). Brucella induces an unfolded protein response via TcpB that supports intracellular replication in macrophages. PLoS Pathog. 9:e1003785. doi: 10.1371/journal.ppat.1003785

Stahl, S., Burkhart, J. M., Hinte, F., Tirosh, B., Mohr, H., Zahedi, R. P., et al. (2013). Cytomegalovirus downregulates IRE1 to repress the unfolded protein response. PLoS Pathog. 9:e1003544. doi: 10.1371/journal.ppat.1003544

Tabas, I., and Ron, D. (2011). Integrating the mechanisms of apoptosis induced by endoplasmic reticulum stress. Nat. Cell Biol. 13, 184-190. doi: 10.1038/ ncb0311-184

Tesh, V. L. (2012). Activation of cell stress response pathways by Shiga toxins. Cell. Microbiol. 14, 1-9. doi: 10.1111/j.1462-5822.2011.01684.x

Walker, M. J., Barnett, T. C., McArthur, J. D., Cole, J. N., Gillen, C. M., Henningham, A., et al. (2014). Disease manifestations and pathogenic mechanisms of group a Streptococcus. Clin. Microbiol. Rev. 27, 264-301. doi: 10.1128/CMR.00101-13

Walter, P., and Ron, D. (2011). The unfolded protein response: from stress pathway to homeostatic regulation. Science 334, 1081-1086. doi: 10.1126/science. 1209038

Watanabe, S., Shimomura, Y., Ubukata, K., Kirikae, T., and Miyoshi-Akiyama, T. (2013). Concomitant regulation of host tissue-destroying virulence factors and carbohydrate metabolism during invasive diseases induced by group $\mathrm{g}$ streptococci. J. Infect. Dis. 208, 1482-1493. doi: 10.1093/infdis/jit353

Woehlbier, U., and Hetz, C. (2011). Modulating stress responses by the UPRosome: a matter of life and death. Trends Biochem. Sci. 36, 329-337. doi: 10.1016/j.tibs.2011.03.001

Yoshida, H. (2007). ER stress and diseases. FEBS J. 274, 630-658. doi: 10.1111/j.1742-4658.2007.05639.x

Zhang, K., and Kaufman, R. J. (2006). The unfolded protein response: a stress signaling pathway critical for health and disease. Neurology 66, S102-S109. doi: 10.1212/01.wnl.0000192306.98198.ec

Zhang, L., and Wang, A. (2012). Virus-induced ER stress and the unfolded protein response. Front. Plant Sci. 3:293. doi: 10.3389/fpls.2012.00293

Conflict of Interest Statement: The authors declare that the research was conducted in the absence of any commercial or financial relationships that could be construed as a potential conflict of interest.

Received: 28 May 2014; paper pending published: 29 June 2014; accepted: 14 July 2014; published online: 04 August 2014.

Citation: Baruch M, Hertzog BB, Ravins M, Anand A, Cheng CY, Biswas D, Tirosh B and Hanski E (2014) Induction of endoplasmic reticulum stress and unfolded protein response constitutes a pathogenic strategy of group A streptococcus. Front. Cell. Infect. Microbiol. 4:105. doi: 10.3389/fcimb.2014.00105

This article was submitted to the journal Frontiers in Cellular and Infection Microbiology.

Copyright (c) 2014 Baruch, Hertzog, Ravins, Anand, Cheng, Biswas, Tirosh and Hanski. This is an open-access article distributed under the terms of the Creative Commons Attribution License (CC BY). The use, distribution or reproduction in other forums is permitted, provided the original author(s) or licensor are credited and that the original publication in this journal is cited, in accordance with accepted academic practice. No use, distribution or reproduction is permitted which does not comply with these terms. 